\title{
A Case of Aortic Valve Annulus Reconstruction with Bovine Pericardial Patch for Artificial Valve Detachment in Behcet's Disease
}

\author{
Ruhua Shen, Yang Wang, Yang Liu, Bing Zhang, Jianjun Ge \\ Department of Cardiovascular Surgery, The First Affiliated Hospital of USTC, Division of Life Sciences and Medicine, \\ University of Science and Technology of China, Hefei, Anhui, China
}

\section{ABSTRACT}

A 36-year-old patient with Behcet's disease was hospitalized because of severe aortic valve regurgitation accompanied by aortic valve neoplasia and perforation of the valve body. In the first operation, we performed aortic valve replacement and ascending aortoplasty. The regular examination after surgery found no obvious regurgitation or discomfort. Four months later, however, the artificial valve fell off the valve frame and the annulus, with severe perivalvular leakage; the range of detachment was about two thirds. In the second operation, we applied a bovine pericardial patch. The aortic valve annulus was reconstructed using the lower edge of the bovine pericardial patch. The upper edge of the patch was sutured to the top of the aortic sinus to strengthen it, and the lateral edge was sutured 3 to $5 \mathrm{~mm}$ from the left coronary opening. The artificial valve was fixed well 6 months after the operation, and no valve detachment was observed. This method of reconstructing an aortic valve annulus with a bovine pericardial patch may be referential for preventing artificial valve detachment in patients with Behcet's disease.

\section{INTRODUCTION}

Behcet's disease (BD) is an inflammatory disease with unknown causes that can affect the aorta and the aortic valve [Suzuki Kurokawa 2004]. Surgical treatment is necessary for some patients with BD-associated cardiovascular complications, but the clinical manifestations of BD are always not typical. Thus many patients cannot be diagnosed before surgery. Meanwhile, the surgical treatment for BD patients can be difficult, especially because of concurrent arteritis, valve and annulus tissue fragility, and infective endocarditis. In addition, postoperative complications of BD can be serious,

Received August 16, 2020; received in revised form September 17, 2020; accepted September 19, 2020.

R. Shen and Y. Wang contributed equally to this work.

Correspondence: Bing Zhang, No. 17 Lujiang Road, Hefei, Anbui 230001, China; 8617756059814 (e-mail: bzhang@mail.ustc.edu.cn); 7ianjun Ge, No. 17 Lujiang Road, Hefei, Anbui 230001, China; 8613605693993 (e-mail: zkdgij@ustc.edu.cn). such as bleeding, valve detachment, and severe paravalvular leakage [Emmi 2019; Farouk 2014]. An accepted surgical technical standard has not been established for cardiovascular complications in BD. In this study, we report a case of aortic valve annulus reconstruction with a bovine pericardial patch for artificial valve detachment in BD.

\section{CASE REPORT}

In June 2019, a 36-year-old Chinese man was admitted to the hospital because of shortness of breath and inability to lie supine. He had a history of recurrent stomatitis and moyamoya disease, with no history of genital ulcers, eye diseases, or fever. A 4/6 diastolic murmur could be heard at the third intercostal space of the left sternal border. The laboratory test results are shown in the Table. The 5-day blood culture was aseptic, and the sputum culture showed normal bacterial growth. Chest computed tomography (CT) showed double lung edema, infectious lesions of both lungs, bilateral pleural effusions, and ascending aorta outline widened to an outer diameter of $46 \mathrm{~mm}$. Echocardiography showed severe aortic valve regurgitation (Figure 1A), left ventricular end-diastolic and end-systolic diameters of 65 and $45 \mathrm{~mm}$, and left ventricular ejection fraction of 0.57 and suggested neoplasia and perforation of the valve body.

Considering the acute cardiac insufficiency and lung infections, cardiotonic, diuretic, and anti-infective treatments were performed. Second chest CT showed a significant improvement, and the absolute value of neutrophils $(6.11 \times 10-9 / \mathrm{L})$ returned to normal before surgery.

During the operation, we found that the aortic wall was thickened by 3 to $5 \mathrm{~mm}$, the annulus was ulcerated at the junction of the right coronary artery and the noncoronary valve, and there was perforation near the right junction of the noncoronary valve. We resected the diseased valve and implanted an aortic mechanical valve (21\# SJ-Jude) by mattress suture. The 48-hour culture of intraoperative excisions was aseptic. After the operation, patients received antibiotic treatment for 4 weeks, and the blood routine was normal on discharge.

After discharge, the patient was reexamined regularly in the outpatient department without especial discomfort. Echocardiography indicated that the artificial valvular frame was fixed well, and no obvious regurgitation was observed around the valve (Figure 1B). But at the fourth month after the operation, the patient suddenly experienced palpitation 
Table. Laboratory Test Results

\begin{tabular}{lccc}
\hline Property & Normal Value & First Hospitalization & Second Hospitalization \\
\hline NT-proBNP $(\mathrm{pg} / \mathrm{mL})$ & 0 to 100 & 2464 & 20,054 \\
CRP $(\mathrm{mg} / \mathrm{L})$ & 0.00 to 8.00 & 43.40 & 29.6 \\
ESR $(\mathrm{mm} / \mathrm{h})$ & 0.00 to 15.00 & 70.00 & 80.00 \\
ANC $(/ \mathrm{L})$ & 2.00 to $7.00 \times 10^{-9}$ & $7.5 \times 10^{-9}$ & $4.22 \times 10^{-9}$ \\
WBC $(/ \mathrm{L})$ & 4.00 to $10.00 \times 10^{-9}$ & $9.81 \times 10^{-9}$ & $6.24 \times 10^{-9}$ \\
\hline
\end{tabular}

ANC indicates absolute neutrophil count; CRP, C reactive protein; ESR, erythrocyte sedimentation rate; NT-proBNP, amino-terminal B-type natriuretic peptide precursor; WBC, white blood cell count.

A

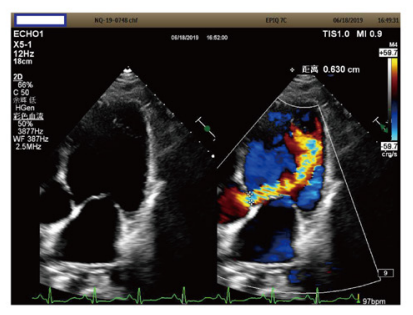

C
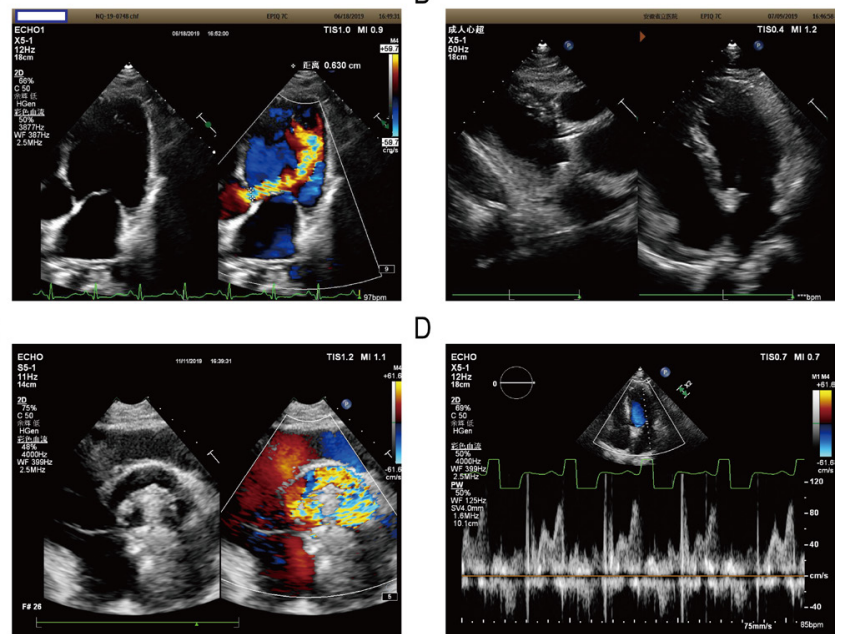

D

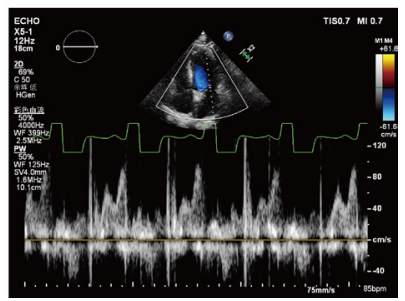

Figure 1. Echocardiography before the first operation (A), 2 months after the first operation (B), before the second operation (C), and 6 months after the second operation (D).

and chest discomfort. The outpatient cardiac ultrasound indicated that the artificial aortic valve was wobbly, the artificial valve was split away from the valve frame and valve annulus (with severe perivalvular leakage), and the range of detachment was about two thirds (Figure 1C and Video). The left ventricular end-diastolic and end-systolic diameters were 64 and $49 \mathrm{~mm}$, respectively. The left ventricular ejection fraction was 0.45 . Laboratory test results are shown in the Table. Surgery was arranged immediately after the admission assessment.

In the second aortic valve replacement, we found no obvious valve annulus tissue under the right and left coronary valves. Only a few fiber bundles were connected to the annulus, and no neoplasia was formed. We chose a bovine pericardial patch (Balance Medical, Beijing, China) to reconstruct and strengthen the aortic valve annulus with prolene line (5-0), using the lower edge of the bovine pericardial patch. The upper edge of the patch was sutured to the top of the aortic sinus to strengthen the aortic sinus, and the lateral edge was sutured 3 to $5 \mathrm{~mm}$ from the left coronary opening. 2-0 Ethibond wire gaskets were chosen

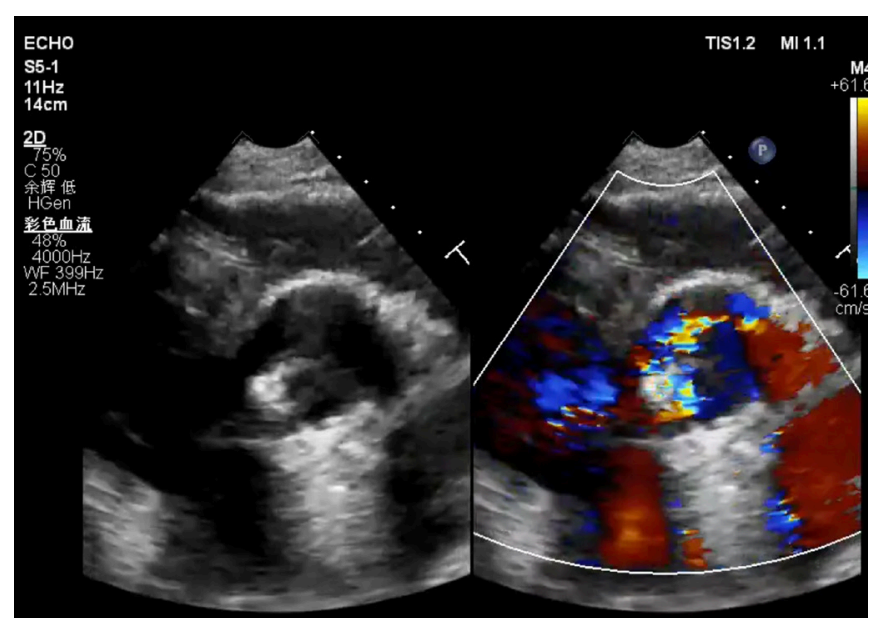

Video. Echocardiography before the second operation. The artificial valve was split away from the valve frame and the annulus with severe perivalvular leakage; the range of detachment was about two thirds. Click here: https://vimeo.com/470671681

to intermittently suture. All the gaskets were placed on the left ventricular surface. The valve annulus was enlarged compared with the anterior; thus a 24\# Medtronic aortic valve was implanted (Figure 2).

After the operation, the patient developed a third-degree conduction block and was discharged from the hospital after a permanent pacemaker was placed. After taking colchicine $(0.5$ $\mathrm{mg}$ bid) for 1 month after surgery, it was changed to methylprednisolone (8 $\mathrm{mg}$ tid) and thalidomide (50 $\mathrm{mg} \mathrm{qn})$. Echocardiography 6 months after the second operation showed that the artificial valve was fixed well, and no obvious regurgitation was seen around the valve (Figure 1D).

\section{DISCUSSION}

$\mathrm{BD}$ is a multisystem disease characterized by recurrent oral and genital ulcers, recurrent uveitis, and skin, mucous membrane, urogenital, neurological, and vascular manifestations [Hatemi 2019]. BD can also cause aortic valve disease and further lead to aortic valve regurgitation. But the effect 
A

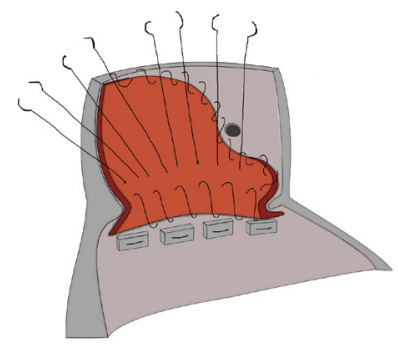

C

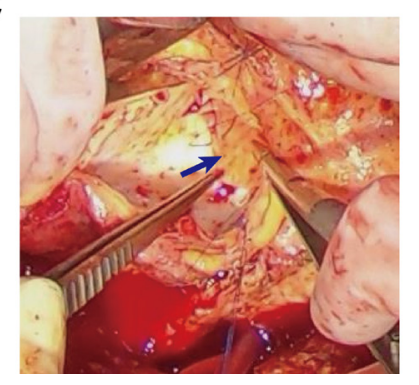

B

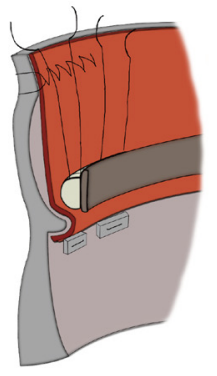

D

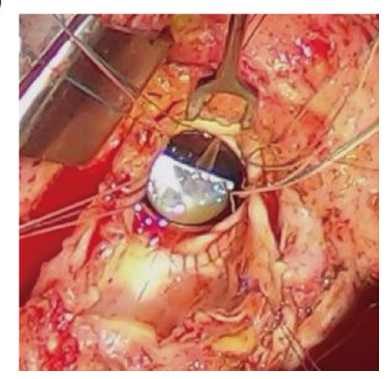

Figure 2. Diagram of the aortic valve annulus reconstruction with bovine pericardial patch. (A) Illustration of the annulus reconstruction with the patch before valve replacement. (B) Replacement of the aortic valve. (C and D) Intraoperative pictures corresponding to A and B, respectively.

of aortic valve replacement is not always ideal, because of common tissue fragility, paravalvular leakage, valve detachment, and pseudoaneurysm formation after the replacement [Ando 2000; Azuma 2009; Tang 2012].

In the active phase of vasculitis, inflammation causes the aortic ring and aortic wall tissue to be fragile, which can lead to prosthetic valve detachment [So 2014]. Through strengthening the aortic valve annulus by clamping the valve annulus in the bovine pericardium, we believe that the rupture stress can be effectively transmitted and distributed to the bovine pericardium, and the vertical gasket suture can further assist the firm fixation of the prosthesis to the ring tissue. At the same time, the aortic sinus is reinforced by the bovine pericardium, which can prevent expansion of the aortic sinus and reduce the formation of pseudoaneurysms.

Strong immunosuppressive therapy can significantly reduce surgical complications and the need for reoperation. In contrast, patients who do not receive immunosuppressive treatment have a high incidence of postoperative complications, such as artificial valve rupture and perivalvular leakage [Yuan 2014]. Because of valve detachment, cardiac function cannot be maintained, and immunosuppressive therapy

cannot be performed before surgery. Thus, postoperative immunosuppressive therapy is necessary.

\section{CONCLUSION}

In summary, this method of reconstructing the aortic valve annulus, using a bovine pericardial patch to treat aortic valve detachment in $\mathrm{BD}$, strengthens the aortic valve annulus and aortic sinus, avoiding mechanical manipulation of the coronary artery and repeat valve detachment.

\section{ACKNOWLEDGEMENTS}

This work was supported by the National Natural Science Foundation of China (grant NSFC 81470530 to J.G.) and the Major Science and Technology Projects of Anhui (18030801132 to J.G.).

\section{REFERENCES}

Ando M, Sasako Y, Okita Y, Tagusari O, Kitamura S. Valved conduit operation for aortic regurgitation associated with Behçet's disease. Jpn J Thorac Cardiovasc Surg 2000;48:424-427.

Azuma T, Yamazaki K, Saito S, Kurosawa H. Aortic valve replacement in Behcet's disease: Surgical modification to prevent valve detachment. Eur J Cardio Thorac Surg 2009;36:771-772.

Emmi G, Bettiol A, Silvestri E, et al. Vascular Behçet's syndrome: An update. Intern Emerg Med 2019;14:645-652.

Farouk H. Behçet's disease, echocardiographers, and cardiac surgeons: Together is better. Echocardiography (Mount Kisco, NY) 2014;31:783-787.

Hatemi G, Seyahi E, Fresko I, Talarico R, Hamuryudan V. One year in review 2019: Behçet's syndrome. Clin Exp Rheumatol 2019;37(suppl 121):3-17.

So H, Yip ML. Acute myocardial infarction and subclavian artery occlusion in a 41-year-old woman with Behçet's disease: Coronary and large vessel arteritis. Singapore Med J 2014;55:e145-e147.

Suzuki Kurokawa M, Suzuki N. Behcet's disease. Clin Exp Med 2004;4:10-20.

Tang Y, Xu Z, Liao Z, Xu J. Supraannular aortic replacement for severe valve detachment attributable to Behçet's disease. Ann Thorac Surg 2012;94:e55-e57.

Yuan SM. Cardiothoracic interventions in Behçet's disease. Clin Exp Rheumatol 2014;32(4 suppl 84):S130-S139. 